\title{
YUKAWA QUASI-UNIFICATION AND INFLATION
}

\author{
G. LAZARIDES AND C. PALLIS \\ Physics Division, School of Technology, \\ Aristotle University of Thessaloniki, \\ 54124 Thessaloniki, GREECE \\ E-mail: lazaride@eng.auth.gr,kpallis@auth.gr
}

\begin{abstract}
We review the construction of a concrete supersymmetric grand unified model, which naturally leads to a moderate violation of 'asymptotic' Yukawa unification and, thus, can allow an acceptable $b$-quark mass within the constrained minimal supersymmetric standard model with $\mu>0$. The model possesses a wide and natural range of parameters which is consistent with the data on the cold dark matter abundance in the universe, $b \rightarrow s \gamma$, the muon anomalous magnetic moment and the Higgs boson masses. Also, it automatically leads to a new version of shifted hybrid inflation, which avoids overproduction of monopoles at the end of inflation by using only renormalizable terms.
\end{abstract}

\section{Introduction}

The most restrictive version of the minimal supersymmetric standard model (MSSM) with gauge coupling unification, radiative electroweak breaking and universal boundary conditions from gravity-mediated soft supersymmetry (SUSY) breaking, known as constrained MSSM (CMSSM) ${ }^{1}$, can be made even more predictive, if we impose Yukawa unification (YU), i.e. assume that the three third generation Yukawa coupling constants unify at the SUSY grand unified theory (GUT) scale, $M_{\mathrm{GUT}}$. The requirement of YU can be achieved by embedding the MSSM in a SUSY GUT with a gauge group containing $\mathrm{SU}(4)_{c}$ and $\mathrm{SU}(2)_{R}$. Indeed, assuming that the electroweak Higgs superfields $h_{1}^{\mathrm{ew}}, h_{2}^{\mathrm{ew}}$ and the third family right handed quark superfields $t^{c}, b^{c}$ form $\mathrm{SU}(2)_{R}$ doublets, we obtain ${ }^{2}$ the 'asymptotic' Yukawa coupling relation $h_{t}=h_{b}$ and, hence, large $\tan \beta \sim m_{t} / m_{b}$. Moreover, if the third generation quark and lepton $\mathrm{SU}(2)_{L}$ doublets [singlets] $q_{3}$ and $l_{3}\left[b^{c}\right.$ and $\left.\tau^{c}\right]$ form a $\mathrm{SU}(4)_{c}$ 4-plet $[\overline{4}$-plet $]$ and the Higgs doublet $h_{1}^{\text {ew }}$ which couples to them is a $\mathrm{SU}(4)_{c}$ singlet, we get $h_{b}=h_{\tau}$ and the 'asymptotic' relation $m_{b}=m_{\tau}$ follows. The simplest GUT gauge group 
which contains both $\mathrm{SU}(4)_{c}$ and $\mathrm{SU}(2)_{R}$ is the Pati-Salam (PS) group $G_{\mathrm{PS}}=\mathrm{SU}(4)_{c} \times \mathrm{SU}(2)_{L} \times \mathrm{SU}(2)_{R}$ and we will use it in our analysis.

However, applying YU in the context of the CMSSM and given the experimental values of the top-quark and tau-lepton masses (which naturally restrict $\tan \beta \simeq 50$ ), the resulting value of the $b$-quark mass turns out to be unacceptable. This is due to the fact that, in the large $\tan \beta$ regime, the tree-level $b$-quark mass receives sizeable SUSY corrections ${ }^{3,4,5,6}$ (about $20 \%$ ), which have the sign of $\mu$ (with the standard sign convention ${ }^{7}$ ) and drive, for $\mu>[<] 0$, the corrected $b$-quark mass at $M_{Z}, m_{b}\left(M_{Z}\right)$, well above [somewhat below] its $95 \%$ confidence level (c.l.) experimental range:

$$
2.684 \mathrm{GeV} \lesssim m_{b}\left(M_{Z}\right) \lesssim 3.092 \mathrm{GeV} \text { with } \alpha_{s}\left(M_{Z}\right)=0.1185 .
$$

This is derived by appropriately ${ }^{8}$ evolving the corresponding range of $m_{b}\left(m_{b}\right)$ in the $\overline{M S}$ scheme (i.e. $\left.3.95-4.55 \mathrm{GeV}\right)$ up to $M_{Z}$ in accordance with Ref. 9. We see that, for both signs of $\mu$, YU leads to an unacceptable $b$-quark mass with the $\mu<0$ case being less disfavored.

A way out of this $m_{b}$ problem can be found ${ }^{8}$ without abandoning the CMSSM (in contrast to the usual strategy ${ }^{6,10,11,12}$ ) or YU altogether. We can rather modestly correct $\mathrm{YU}$ by including an extra $\mathrm{SU}(4)_{c}$ non-singlet Higgs superfield with Yukawa couplings to the quarks and leptons. The Higgs $\mathrm{SU}(2)_{L}$ doublets contained in this superfield can naturally develop ${ }^{13}$ subdominant vacuum expectation values (VEVs) and mix with the main electroweak doublets, which are assumed to be $\mathrm{SU}(4)_{c}$ singlets and form a $\mathrm{SU}(2)_{R}$ doublet. This mixing can, in general, violate $\mathrm{SU}(2)_{R}$. Consequently, the resulting electroweak Higgs doublets $h_{1}^{\text {ew }}, h_{2}^{\text {ew }}$ do not form a $\mathrm{SU}(2)_{R}$ doublet and also break $\mathrm{SU}(4)_{c}$. The required deviation from $\mathrm{YU}$ is expected to be more pronounced for $\mu>0$. Despite this, we will study here this case, since the $\mu<0$ case has been excluded ${ }^{14}$ by combining the Wilkinson microwave anisotropy probe (WMAP) restrictions ${ }^{15}$ on the cold dark matter $(\mathrm{CDM})$ in the universe with the recent experimental results ${ }^{16}$ on the inclusive branching ratio $\mathrm{BR}(b \rightarrow s \gamma)$. The same SUSY GUT model which, for $\mu>0$ and universal boundary conditions, remedies the $m_{b}$ problem leads to a new version ${ }^{17}$ of shifted hybrid inflation ${ }^{18}$, which avoids monopole overproduction at the end of inflation and, in contrast to the older version ${ }^{18}$, is based only on renormalizable interactions.

In Sec. 2, we review the construction of a SUSY GUT model which modestly violates $\mathrm{YU}$, yielding an appropriate Yukawa quasi-unification condition (YQUC), which is derived in Sec. 3. We then describe the resulting CMSSM in Sec. 4 and introduce the various cosmological and phenomeno- 
logical requirements which restrict its parameter space in Sec. 5. In Sec. 6, we delineate the allowed range of parameters for $\mu>0$ and, in Sec. 7, we outline the corresponding inflationary scenario. Finally, in Sec. 8, we summarize our conclusions.

\section{The SUSY GUT Model}

We will take the SUSY GUT model of shifted hybrid inflation ${ }^{18}$ (see also Ref. 19) as our starting point. It is based on $G_{\mathrm{PS}}$, which is the simplest gauge group that can lead to YU. The representations under $G_{\mathrm{PS}}$ and the global charges of the various matter and Higgs superfields contained in this model are presented in Table 1, which also contains the extra Higgs superfields required for accommodating an adequate violation of $\mathrm{YU}$ (see below). The matter superfields are $F_{i}$ and $F_{i}^{c}(i=1,2,3)$, while the electroweak Higgs doublets belong to the superfield $h$. So, all the requirements for exact YU are fulfilled. The breaking of $G_{\mathrm{PS}}$ down to the standard model (SM) gauge group $G_{\mathrm{SM}}$ is achieved by the superheavy VEVs $\left(\sim M_{\mathrm{GUT}}\right)$ of the right handed neutrino type components of a conjugate pair of Higgs superfields $H^{c}, \bar{H}^{c}$. The model also contains a gauge singlet $S$ which triggers the breaking of $G_{\mathrm{PS}}$, a $\mathrm{SU}(4)_{c}$ 6-plet $G$ which gives ${ }^{20}$ masses to the right handed down quark type components of $H^{c}, \bar{H}^{c}$, and a pair of gauge singlets $N, \bar{N}$ for solving ${ }^{21}$ the $\mu$ problem of the MSSM via a Peccei-Quinn (PQ) symmetry. In addition to $G_{\mathrm{PS}}$, the model possesses two global U(1) symmetries, namely a $\mathrm{R}$ and a $\mathrm{PQ}$ symmetry, as well as a discrete $Z_{2}^{\mathrm{mp}}$ symmetry ('matter parity').

A moderate violation of exact YU can be naturally accommodated in this model by adding a new Higgs superfield $h^{\prime}$ with Yukawa couplings $F F^{c} h^{\prime}$. Actually, $(\mathbf{1 5 , 2 , 2})$ is the only representation, besides $(\mathbf{1}, \mathbf{2}, \mathbf{2})$, which possesses such couplings to the fermions. In order to give superheavy masses to the color non-singlet components of $h^{\prime}$, we need to include one more Higgs superfield $\bar{h}^{\prime}$ with the superpotential coupling $\bar{h}^{\prime} h^{\prime}$, whose coefficient is of the order of $M_{\mathrm{GUT}}$.

After the breaking of $G_{\mathrm{PS}}$ to $G_{\mathrm{SM}}$, the two color singlet $\mathrm{SU}(2)_{L}$ doublets $h_{1}^{\prime}, h_{2}^{\prime}$ contained in $h^{\prime}$ can mix with the corresponding doublets $h_{1}, h_{2}$ in $h$. This is mainly due to the terms $\bar{h}^{\prime} h^{\prime}$ and $H^{c} \bar{H}^{c} \bar{h}^{\prime} h$. Actually, since

$$
\begin{aligned}
& H^{c} \bar{H}^{c}=(\overline{\mathbf{4}}, \mathbf{1}, \mathbf{2})(\mathbf{4}, \mathbf{1}, \mathbf{2})=(\mathbf{1 5}, \mathbf{1}, \mathbf{1}+\mathbf{3})+\cdots, \\
& \bar{h}^{\prime} h=(\mathbf{1 5}, \mathbf{2}, \mathbf{2})(\mathbf{1}, \mathbf{2}, \mathbf{2})=(\mathbf{1 5}, \mathbf{1}, \mathbf{1}+\mathbf{3})+\cdots,
\end{aligned}
$$

there are two independent couplings of the type $H^{c} \bar{H}^{c} \bar{h}^{\prime} h$ (both suppressed by the string scale $M_{\mathrm{S}} \approx 5 \times 10^{17} \mathrm{GeV}$, being non-renormalizable). One of 
Table 1. Superfield Content of the Model

\begin{tabular}{|c|c|c|c|c|}
\hline \multirow[t]{2}{*}{ Superfields } & \multirow[t]{2}{*}{$\begin{array}{l}\text { Representations } \\
\text { under } G_{\mathrm{PS}}\end{array}$} & \multicolumn{3}{|c|}{$\begin{array}{c}\text { Global } \\
\text { Symmetries }\end{array}$} \\
\hline & & $R$ & $P Q$ & $Z_{2}^{\mathrm{mp}}$ \\
\hline \multicolumn{5}{|c|}{ Matter Fields } \\
\hline$F_{i}$ & $(4,2,1)$ & $1 / 2$ & -1 & 1 \\
\hline$F_{i}^{c}$ & $(\overline{4}, 1,2)$ & $1 / 2$ & 0 & -1 \\
\hline \multicolumn{5}{|c|}{ Higgs Fields } \\
\hline$h$ & $(1,2,2)$ & 0 & 1 & 0 \\
\hline$H^{c}$ & $(\overline{4}, \mathbf{1}, \mathbf{2})$ & 0 & 0 & 0 \\
\hline $\bar{H}^{c}$ & $(4,1,2)$ & 0 & 0 & 0 \\
\hline$S$ & $(\mathbf{1}, \mathbf{1}, \mathbf{1})$ & 1 & 0 & 0 \\
\hline$G$ & $(\mathbf{6}, \mathbf{1}, \mathbf{1})$ & 1 & 0 & 0 \\
\hline$N$ & $(\mathbf{1}, \mathbf{1}, \mathbf{1})$ & $1 / 2$ & -1 & 0 \\
\hline $\bar{N}$ & $(\mathbf{1}, \mathbf{1}, \mathbf{1})$ & 0 & 1 & 0 \\
\hline \multicolumn{5}{|c|}{ Extra Higgs Fields } \\
\hline$h^{\prime}$ & $(15,2,2)$ & 0 & 1 & 0 \\
\hline $\bar{h}^{\prime}$ & $(15,2,2)$ & 1 & -1 & 0 \\
\hline$\phi$ & $(15,1,3)$ & 0 & 0 & 0 \\
\hline $\bar{\phi}$ & $(\mathbf{1 5}, \mathbf{1}, \mathbf{3})$ & 1 & 0 & 0 \\
\hline
\end{tabular}

them is between the $\mathrm{SU}(2)_{R}$ singlets in $H^{c} \bar{H}^{c}$ and $\bar{h}^{\prime} h$, and the other between the $\mathrm{SU}(2)_{R}$ triplets in these combinations. So, we obtain two bilinear terms $\bar{h}_{1}^{\prime} h_{1}$ and $\bar{h}_{2}^{\prime} h_{2}$ with different coefficients, which are suppressed by $M_{\mathrm{GUT}} / M_{\mathrm{S}}$. These terms together with the terms $\bar{h}_{1}^{\prime} h_{1}^{\prime}$ and $\bar{h}_{2}^{\prime} h_{2}^{\prime}$ from $\bar{h}^{\prime} h^{\prime}$, which have equal coefficients, generate different mixings between $h_{1}, h_{1}^{\prime}$ and $h_{2}, h_{2}^{\prime}$. Consequently, the resulting electroweak doublets $h_{1}^{\mathrm{ew}}, h_{2}^{\mathrm{ew}}$ contain $\mathrm{SU}(4)_{c}$ violating components suppressed by $M_{\mathrm{GUT}} / M_{\mathrm{S}}$ and fail to form a $\mathrm{SU}(2)_{R}$ doublet by an equally suppressed amount. So, YU is moderately violated. Unfortunately, as it turns out, this violation is not adequate for correcting the $b$-quark mass within the CMSSM for $\mu>0$.

In order to allow for a more sizable violation of YU, we further extend the model by including $\phi$ with the coupling $\phi \bar{h}^{\prime} h$. To give superheavy masses to the color non-singlets in $\phi$, we introduce one more superfield $\bar{\phi}$ with the coupling $\bar{\phi} \phi$, whose coefficient is of order $M_{\mathrm{GUT}}$. 
The terms $\bar{\phi} \phi$ and $\bar{\phi} H^{c} \bar{H}^{c}$ imply that, after the breaking of $G_{\mathrm{PS}}$ to $G_{\mathrm{SM}}$, $\phi$ acquires a superheavy VEV of order $M_{\mathrm{GUT}}$. The coupling $\phi \bar{h}^{\prime} h$ then generates $\mathrm{SU}(2)_{R}$ violating unsuppressed bilinear terms between the doublets in $\bar{h}^{\prime}$ and $h$. These terms can certainly overshadow the corresponding ones from the non-renormalizable term $H^{c} \bar{H}^{c} \bar{h}^{\prime} h$. The resulting $\mathrm{SU}(2)_{R}$ violating mixing of the doublets in $h$ and $h^{\prime}$ is then unsuppressed and we can obtain stronger violation of YU.

\section{The Yukawa Quasi-Unification Condition}

To further analyze the mixing of the doublets in $h$ and $h^{\prime}$, observe that the part of the superpotential corresponding to the symbolic couplings $\bar{h}^{\prime} h^{\prime}$, $\phi \bar{h}^{\prime} h$ is properly written as

$$
m \operatorname{tr}\left(\bar{h}^{\prime} \epsilon \tilde{h}^{\prime} \epsilon\right)+p \operatorname{tr}\left(\bar{h}^{\prime} \epsilon \phi \tilde{h} \epsilon\right),
$$

where $\epsilon \epsilon$ is the antisymmetric $2 \times 2$ matrix with $\epsilon_{12}=+1$, tr denotes trace taken with respect to the $\mathrm{SU}(4)_{c}$ and $\mathrm{SU}(2)_{L}$ indices and tilde denotes the transpose of a matrix.

After the breaking of $G_{\mathrm{PS}}$ to $G_{\mathrm{SM}}, \phi$ acquires a VEV $\langle\phi\rangle \sim M_{\mathrm{GUT}}$. Substituting it by this VEV in the above couplings, we obtain

$$
\begin{aligned}
& \operatorname{tr}\left(\bar{h}^{\prime} \epsilon \tilde{h}^{\prime} \epsilon\right)=\tilde{\bar{h}}_{1}^{\prime} \epsilon h_{2}^{\prime}+\tilde{h}_{1}^{\prime} \epsilon \bar{h}_{2}^{\prime}+\cdots, \\
& \operatorname{tr}\left(\bar{h}^{\prime} \epsilon \phi \tilde{h} \epsilon\right)=\frac{\langle\phi\rangle}{\sqrt{2}} \operatorname{tr}\left(\bar{h}^{\prime} \epsilon \sigma_{3} \tilde{h} \epsilon\right)=\frac{\langle\phi\rangle}{\sqrt{2}}\left(\tilde{\bar{h}}_{1}^{\prime} \epsilon h_{2}-\tilde{h}_{1} \epsilon \bar{h}_{2}^{\prime}\right),
\end{aligned}
$$

where the ellipsis in Eq. (3) contains the colored components of $\bar{h}^{\prime}, h^{\prime}$ and $\sigma_{3}=\operatorname{diag}(1,-1)$. Inserting Eqs. (3) and (4) into Eq. (2), we obtain

$$
m \tilde{\bar{h}}_{1}^{\prime} \epsilon\left(h_{2}^{\prime}-\alpha_{1} h_{2}\right)+m\left(\tilde{h}_{1}^{\prime}+\alpha_{1} \tilde{h}_{1}\right) \epsilon \bar{h}_{2}^{\prime} \text { with } \alpha_{1}=-p\langle\phi\rangle / \sqrt{2} m .
$$

So, we get two pairs of superheavy doublets with mass $m$. They are predominantly given by

$$
\bar{h}_{1}^{\prime}, \frac{h_{2}^{\prime}-\alpha_{1} h_{2}}{\sqrt{1+\left|\alpha_{1}\right|^{2}}} \text { and } \frac{h_{1}^{\prime}+\alpha_{1} h_{1}}{\sqrt{1+\left|\alpha_{1}\right|^{2}}}, \bar{h}_{2}^{\prime} .
$$

The orthogonal combinations of $h_{1}, h_{1}^{\prime}$ and $h_{2}, h_{2}^{\prime}$ constitute the electroweak doublets

$$
h_{1}^{\mathrm{ew}}=\frac{h_{1}-\alpha_{1}^{*} h_{1}^{\prime}}{\sqrt{1+\left|\alpha_{1}\right|^{2}}} \text { and } h_{2}^{\mathrm{ew}}=\frac{h_{2}+\alpha_{1}^{*} h_{2}^{\prime}}{\sqrt{1+\left|\alpha_{1}\right|^{2}}} .
$$

The superheavy doublets in Eq. (6) must have vanishing VEVs, which readily implies that $\left\langle h_{1}^{\prime}\right\rangle=-\alpha_{1}\left\langle h_{1}\right\rangle,\left\langle h_{2}^{\prime}\right\rangle=\alpha_{1}\left\langle h_{2}\right\rangle$. Equation (7) then 
gives $\left\langle h_{1}^{\text {ew }}\right\rangle=\left(1+\left|\alpha_{1}\right|^{2}\right)^{1 / 2}\left\langle h_{1}\right\rangle,\left\langle h_{2}^{\text {ew }}\right\rangle=\left(1+\left|\alpha_{1}\right|^{2}\right)^{1 / 2}\left\langle h_{2}\right\rangle$. From the third generation Yukawa couplings $y_{33} F_{3} h F_{3}^{c}, 2 y_{33}^{\prime} F_{3} h^{\prime} F_{3}^{c}$, we obtain

$$
\begin{aligned}
& m_{t}=\left|y_{33}\left\langle h_{2}\right\rangle+y_{33}^{\prime}\left\langle h_{2}^{\prime}\right\rangle\right|=\left|\frac{1+\rho \alpha_{1} / \sqrt{3}}{\sqrt{1+\left|\alpha_{2}\right|^{2}}} y_{33}\left\langle h_{2}^{\mathrm{ew}}\right\rangle\right|, \\
& m_{b}=\left|\frac{1-\rho \alpha_{1} / \sqrt{3}}{\sqrt{1+\left|\alpha_{1}\right|^{2}}} y_{33}\left\langle h_{1}^{\mathrm{ew}}\right\rangle\right|, m_{\tau}=\left|\frac{1+\sqrt{3} \rho \alpha_{1}}{\sqrt{1+\left|\alpha_{1}\right|^{2}}} y_{33}\left\langle h_{1}^{\mathrm{ew}}\right\rangle\right| .
\end{aligned}
$$

where $\rho=y_{33}^{\prime} / y_{33}$. From Eqs. (8) and (9), we see that YU is now replaced by the YQUC,

$$
h_{t}: h_{b}: h_{\tau}=(1+c):(1-c):(1+3 c) \text {, with } 0<c=\rho \alpha_{1} / \sqrt{3}<1 .
$$

For simplicity, we restricted ourselves to real values of $c$ only which lie between zero and unity.

\section{The resulting CMSSM}

Below $M_{\mathrm{GUT}}$, the particle content of our model reduces to this of MSSM (modulo SM singlets). We assume universal soft SUSY breaking scalar masses $m_{0}$, gaugino masses $M_{1 / 2}$ and trilinear scalar couplings $A_{0}$ at $M_{\mathrm{GUT}}$. Therefore, the resulting MSSM is the so-called CMSSM ${ }^{1}$ with $\mu>0$ and supplemented by Eq. (10). With these initial conditions, we run the MSSM renormalization group equations (RGEs) ${ }^{22}$ between $M_{\mathrm{GUT}}$ and a common SUSY threshold $M_{\mathrm{SUSY}} \simeq\left(m_{\tilde{t}_{1}} m_{\tilde{t}_{2}}\right)^{1 / 2}\left(\tilde{t}_{1,2}\right.$ are the stop mass eigenstates $)$ determined in consistency with the SUSY spectrum. At $M_{\mathrm{SUSY}}$, we impose radiative electroweak symmetry breaking, evaluate the SUSY spectrum and incorporate the SUSY corrections ${ }^{4,5,6}$ to the $b$ and $\tau$ masses. Note that the corrections to the $\tau$ mass (almost $4 \%$ ) lead ${ }^{14}$ to a small reduction of $\tan \beta$. From $M_{\mathrm{SUSY}}$ to $M_{Z}$, the running of gauge and Yukawa coupling constants is continued using the SM RGEs.

For presentation purposes, $M_{1 / 2}$ and $m_{0}$ can be replaced ${ }^{22}$ by the lightest SUSY particle (LSP) mass, $m_{\mathrm{LSP}}$, and the relative mass splitting between this particle and the lightest stau $\tilde{\tau}_{2}, \Delta_{\tilde{\tau}_{2}}=\left(m_{\tilde{\tau}_{2}}-m_{\mathrm{LSP}}\right) / m_{\mathrm{LSP}}$. For simplicity, we restrict this presentation to the $A_{0}=0$ case (for $A_{0} \neq 0$ see Refs. 8 and 23). So, our input parameters are $m_{\text {LSP }}$ and $\Delta_{\tilde{\tau}_{2}}$.

For any given $m_{b}\left(M_{Z}\right)$ in the range in Eq. (1) and with fixed $m_{t}\left(m_{t}\right)=$ $166 \mathrm{GeV}$ and $m_{\tau}\left(M_{Z}\right)=1.746 \mathrm{GeV}$, we can determine the parameters $c$ and $\tan \beta$ at $M_{\mathrm{SUSY}}$ so that the YQUC in Eq. (10) is satisfied. 


\section{Cosmological and Phenomenological Constraints}

Restrictions on the parameters of our model can be derived by imposing a number of cosmological and phenomenological requirements (for similar recent analyses, see Refs. 11, 12 and 24). These constraints result from:

- Cold dark matter considerations. In the context of CMSSM, the LSP can be the lightest neutralino. It naturally arises ${ }^{25}$ as a CDM candidate. We require its relic abundance, $\Omega_{\mathrm{LSP}} h^{2}$, not to exceed the $95 \%$ c.l. upper bound on the CDM abundance derived ${ }^{15}$ by WMAP:

$$
\Omega_{\mathrm{CDM}} h^{2} \lesssim 0.13
$$

We calculate $\Omega_{\mathrm{LSP}} h^{2}$ using micrOMEGAs ${ }^{26}$, which is certainly one of the most complete publicly available codes. It includes all possible coannihilation processes ${ }^{27}$ and one-loop QCD corrections ${ }^{28}$ to the Higgs decay widths and couplings to fermions.

- Branching ratio of $b \rightarrow s \gamma$. Taking into account the recent experimental results ${ }^{16}$ on this ratio, $\mathrm{BR}(b \rightarrow s \gamma)$, and combining ${ }^{8}$ appropriately the experimental and theoretical errors involved, we obtain the following $95 \%$ c.l. range:

$$
1.9 \times 10^{-4} \lesssim \mathrm{BR}(b \rightarrow s \gamma) \lesssim 4.6 \times 10^{-4} .
$$

We compute $\mathrm{BR}(b \rightarrow s \gamma)$ by using an updated version of the relevant calculation contained in the micrOMEGAs package ${ }^{26}$. In this code, the SM contribution is calculated following Ref. 29. The charged Higgs $\left(H^{ \pm}\right)$contribution is evaluated by including the next-to-leading order (NLO) QCD corrections $^{30}$ and $\tan \beta$ enhanced contributions ${ }^{30}$. The dominant SUSY contribution includes resummed NLO SUSY QCD corrections ${ }^{30}$, which hold for large $\tan \beta$.

- Muon anomalous magnetic moment. The deviation, $\delta a_{\mu}$, of the measured value of $a_{\mu}$ from its predicted value in the SM, $a_{\mu}^{\mathrm{SM}}$, can be attributed to SUSY contributions, calculated by using the micrOMEGAs routine ${ }^{31}$. The calculation of $a_{\mu}^{\mathrm{SM}}$ is not yet stabilized mainly because of the instability of the hadronic vacuum polarization contribution. According to the most up-to-date evaluation ${ }^{32}$, there is still a considerable discrepancy between the findings based on the $e^{+} e^{-}$annihilation data and the ones based on the $\tau$-decay data. Taking into account these results and the experimental measurement ${ }^{33}$ of $a_{\mu}$, we get the following $95 \%$ c.l. ranges:

$$
\begin{aligned}
&-0.53 \times 10^{-10} \lesssim \delta a_{\mu} \lesssim 44.7 \times 10^{-10}, \quad e^{+} e^{-} \text {-based } \\
&-13.6 \times 10^{-10} \lesssim \delta a_{\mu} \lesssim 28.4 \times 10^{-10}, \quad \tau \text {-based } .
\end{aligned}
$$




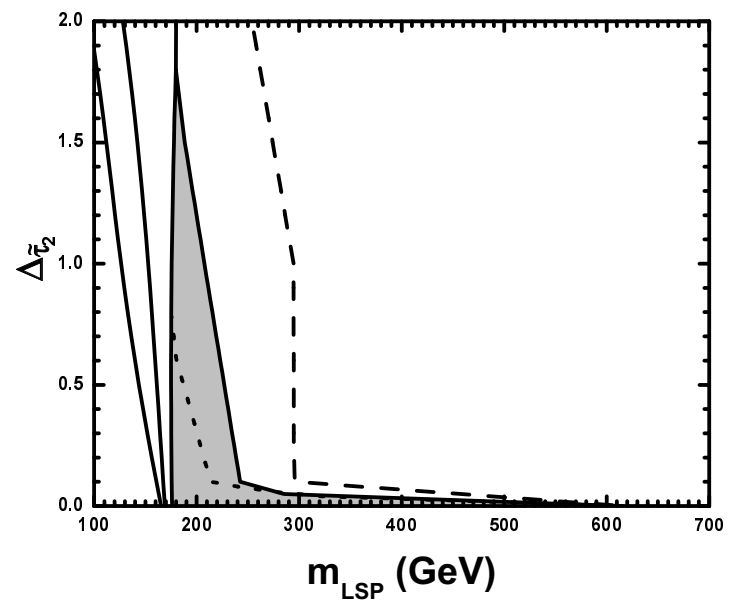

Figure 1. The various restrictions on the $m_{\mathrm{LSP}}-\Delta_{\tilde{\tau}_{2}}$ plane for $\mu>0, A_{0}=0$ and $\alpha_{s}\left(M_{Z}\right)=0.1185$. From left to right, the solid lines depict the lower bounds on $m_{\mathrm{LSP}}$ from $\delta a_{\mu}<44.7 \times 10^{-10}, \operatorname{BR}(b \rightarrow s \gamma)>1.9 \times 10^{-4}$ and $m_{h}>114.4 \mathrm{GeV}$ and the upper bound on $m_{\mathrm{LSP}}$ from $\Omega_{\mathrm{LSP}} h^{2}<0.13$ for $m_{b}\left(M_{Z}\right)=2.888 \mathrm{GeV}$. The dashed [dotted] line depicts the bound on $m_{\mathrm{LSP}}$ from $\Omega_{\mathrm{LSP}} h^{2}<0.13$ for $m_{b}\left(M_{Z}\right)=2.684$ [3.092] GeV. The allowed area for $m_{b}\left(M_{Z}\right)=2.888 \mathrm{GeV}$ is shaded.

Following the common practice ${ }^{24}$, we adopt the restrictions to parameters induced by Eq. (13), since Eq. (14) is considered as quite oracular, due to poor $\tau$-decay data.

- Collider bounds. Here, the only relevant collider bound is the $95 \%$ c.l. LEP bound ${ }^{34}$ on the mass of the lightest CP-even neutral Higgs boson $h$ :

$$
m_{h} \gtrsim 114.4 \mathrm{GeV} \text {. }
$$

The SUSY corrections to $m_{h}$ are calculated at two loops by using the FeynHiggsFast program ${ }^{35}$ included in the micrOMEGAs code ${ }^{26}$.

\section{The Allowed Parameter Space}

We will now try to delineate the parameter space of our model with $\mu>0$ which is consistent with the constraints in Sec. 5. The restrictions on the $m_{\mathrm{LSP}}-\Delta_{\tilde{\tau}_{2}}$ plane, for $A_{0}=0$ and the central values of $\alpha_{s}\left(M_{Z}\right)=0.1185$ and $m_{b}\left(M_{Z}\right)=2.888 \mathrm{GeV}$, are indicated in Fig. 1 by solid lines, while the upper bound on $m_{\mathrm{LSP}}$ from Eq. (11), for $m_{b}\left(M_{Z}\right)=2.684$ [3.092] GeV, is depicted by a dashed [dotted] line. We observe the following: 


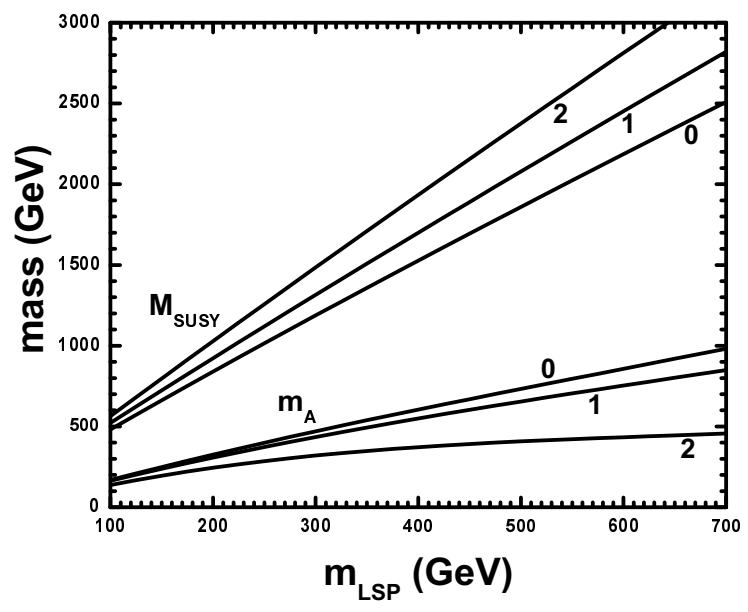

Figure 2. The mass parameters $m_{A}$ and $M_{\text {SUSY }}$ as functions of $m_{\mathrm{LSP}}$ for various values of $\Delta_{\tilde{\tau}_{2}}$, which are indicated on the curves. We take $\mu>0, A_{0}=0, m_{b}\left(M_{Z}\right)=2.888 \mathrm{GeV}$ and $\alpha_{s}\left(M_{Z}\right)=0.1185$.

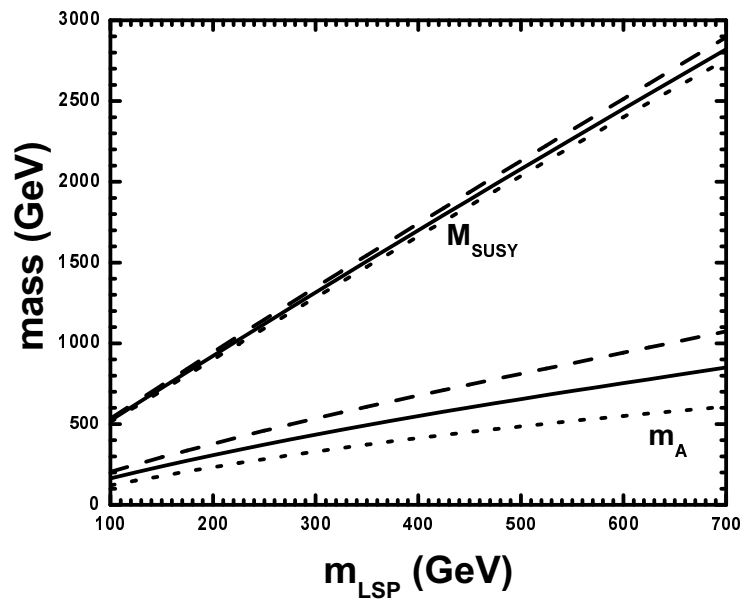

Figure 3. The mass parameters $m_{A}$ and $M_{\mathrm{SUSY}}$ versus $m_{\mathrm{LSP}}$ for $\mu>0, A_{0}=0$, $\Delta_{\tilde{\tau}_{2}}=1, \alpha_{s}\left(M_{Z}\right)=0.1185$ and with $m_{b}\left(M_{Z}\right)=2.684 \mathrm{GeV}$ (dashed lines), $3.092 \mathrm{GeV}$ (dotted lines) or $2.888 \mathrm{GeV}$ (solid lines). 
- The lower bounds on $m_{\mathrm{LSP}}$ are not so sensitive to the variations of $m_{b}\left(M_{Z}\right)$.

- The lower bound on $m_{\mathrm{LSP}}$ from Eq. (15) overshadows all others.

- The upper bound on $m_{\mathrm{LSP}}$ from Eq. (11) is very sensitive to the variations of $m_{b}\left(M_{Z}\right)$. In particular, one notices the extreme sensitivity of the almost vertical part of the corresponding line, where the LSP annihilation via an $A$-boson exchange in the $s$-channel is ${ }^{36}$ by far the dominant process, since $m_{A}$, which is smaller than $2 m_{\mathrm{LSP}}$, is always very close to it as seen from Fig. 2. This sensitivity can be understood from Fig. 3, where $m_{A}$ is depicted versus $m_{\mathrm{LSP}}$ for various $m_{b}\left(M_{Z}\right)$ 's. We see that, as $m_{b}\left(M_{Z}\right)$ decreases, $m_{A}$ increases and approaches $2 m_{\mathrm{LSP}}$. The $A$-pole annihilation is then enhanced and $\Omega_{\mathrm{LSP}} h^{2}$ is drastically reduced causing an increase of the upper bound on $m_{\mathrm{LSP}}$.

- For $\Delta_{\tilde{\tau}_{2}}<0.25$, bino-stau coannihilations ${ }^{27}$ take over leading to a very pronounced reduction of $\Omega_{\mathrm{LSP}} h^{2}$, thereby enhancing the upper limit on $m_{\mathrm{LSP}}$.

For $\mu>0, \alpha_{s}\left(M_{Z}\right)=0.1185$ and $m_{b}\left(M_{Z}\right)=2.888 \mathrm{GeV}$, we find the following allowed ranges of parameters:

$$
\begin{aligned}
& 176 \mathrm{GeV} \lesssim m_{\mathrm{LSP}} \lesssim 615 \mathrm{GeV}, \quad 0 \lesssim \Delta_{\tilde{\tau}_{2}} \lesssim 1.8 \\
& 58 \lesssim \tan \beta \lesssim 59, \quad 0.14 \lesssim c \lesssim 0.17 .
\end{aligned}
$$

\section{The Inflationary Scenario}

One of the most promising inflationary scenarios is hybrid inflation ${ }^{37}$, which uses two real scalars: one which provides the vacuum energy for inflation and a second which is the slowly varying field during inflation. This scheme is naturally incorporated ${ }^{38}$ in SUSY GUTs, but in its standard realization has the following problem ${ }^{39}$ : if the GUT gauge symmetry breaking predicts monopoles (and this is the case of $G_{\mathrm{PS}}$ ), they are copiously produced at the end of inflation leading to a cosmological catastrophe ${ }^{40}$. One way to remedy this is to generate a shifted inflationary trajectory, so that $G_{\mathrm{PS}}$ is already broken during inflation. This could be achieved ${ }^{18}$ in our SUSY GUT model even before the introduction of the extra Higgs superfields, but only by utilizing non-renormalizable terms. However, the introduction of $\phi$ and $\bar{\phi}$ very naturally gives rise ${ }^{17}$ to a shifted inflationary path with the use of renormalizable interactions only. 


\subsection{The Shifted Inflationary Path}

The superpotential terms which are relevant for inflation are given by

$$
W=\kappa S\left(H^{c} \bar{H}^{c}-M^{2}\right)-\beta S \phi^{2}+m \bar{\phi} \phi+\lambda \bar{\phi} H^{c} \bar{H}^{c},
$$

where $M, m \sim M_{\mathrm{GUT}} \simeq 2.86 \times 10^{16} \mathrm{GeV}$, and $\kappa, \beta$ and $\lambda$ are dimensionless coupling constants with $M, m, \kappa, \lambda>0$ by field redefinitions. For simplicity, we take $\beta>0$. (The parameters are normalized so that they correspond to the couplings between the SM singlet components of the superfields.)

The scalar potential obtained from $W$ is given by

$$
\begin{gathered}
V=\left|\kappa\left(H^{c} \bar{H}^{c}-M^{2}\right)-\beta \phi^{2}\right|^{2}+|2 \beta S \phi-m \bar{\phi}|^{2}+\left|m \phi+\lambda H^{c} \bar{H}^{c}\right|^{2} \\
+|\kappa S+\lambda \bar{\phi}|^{2}\left(\left|H^{c}\right|^{2}+\left|\bar{H}^{c}\right|^{2}\right)+\mathrm{D}-\text { terms. }
\end{gathered}
$$

Vanishing of the D-terms yields $\bar{H}^{c *}=e^{i \vartheta} H^{c}\left(H^{c}, \bar{H}^{c}\right.$ lie in their right handed neutrino directions). We restrict ourselves to the direction with $\vartheta=0$ which contains the shifted inflationary path and the SUSY vacua (see below). Performing appropriate $\mathrm{R}$ and gauge transformations, we bring $S$, $H^{c}$ and $\bar{H}^{c}$ to the positive real axis.

From the potential in Eq. (18), we find that the SUSY vacuum lies at

$$
\frac{H^{c} \bar{H}^{c}}{M^{2}} \equiv\left(\frac{v_{0}}{M}\right)^{2}=\frac{1}{2 \xi}(1-\sqrt{1-4 \xi}), \quad \frac{\phi}{M}=-\sqrt{\frac{\kappa \xi}{\beta}}\left(\frac{v_{0}}{M}\right)^{2}
$$

with $S=0$ and $\bar{\phi}=0$, where $\xi=\beta \lambda^{2} M^{2} / \kappa m^{2}<1 / 4$. The potential possesses a shifted flat direction (besides the trivial one) at

$$
\frac{H^{c} \bar{H}^{c}}{M^{2}} \equiv\left(\frac{v}{M}\right)^{2}=\frac{2 \kappa^{2}\left(\frac{1}{4 \xi}+1\right)+\frac{\lambda^{2}}{\xi}}{2\left(\kappa^{2}+\lambda^{2}\right)}, \quad \frac{\phi}{M}=-\frac{1}{2} \sqrt{\frac{\kappa}{\beta \xi}}, \quad \bar{\phi}=-\frac{\kappa}{\lambda} S
$$

with $S>0$ and a constant potential energy density $V_{0}$ given by

$$
\frac{V_{0}}{M^{4}}=\frac{\kappa^{2} \lambda^{2}}{\kappa^{2}+\lambda^{2}}\left(\frac{1}{4 \xi}-1\right)^{2},
$$

which can be used as inflationary path. $V_{0} \neq 0$ breaks SUSY on this path, while the constant non-zero values of $H^{c}, \bar{H}^{c}$ break the GUT gauge symmetry too. The SUSY breaking implies the existence of one-loop radiative corrections ${ }^{41}$ which lift the classical flatness of this path, yielding the necessary inclination for driving the inflaton towards the SUSY vacuum.

The one-loop radiative corrections to $V$ along the shifted inflationary trajectory are calculated by using the Coleman-Weinberg formula ${ }^{42}$ :

$$
\Delta V=\frac{1}{64 \pi^{2}} \sum_{i}(-)^{F_{i}} M_{i}^{4} \ln \frac{M_{i}^{2}}{\Lambda^{2}}
$$


where the sum extends over all helicity states $i, F_{i}$ and $M_{i}^{2}$ are the fermion number and mass squared of the $i$ th state and $\Lambda$ is a renormalization mass scale. In order to use this formula for creating a logarithmic slope which drives the canonically normalized real inflaton field $\sigma=\sqrt{2\left(\kappa^{2}+\lambda^{2}\right)} S / \lambda$ towards the minimum, one has first to derive the mass spectrum of the model on the shifted inflationary path. This is a quite complicated task and we will skip it here.

\subsection{Inflationary Observables}

The slow roll parameters are given by (see e.g. Ref. 43)

$$
\varepsilon \simeq \frac{m_{\mathrm{P}}^{2}}{2}\left(\frac{V^{\prime}(\sigma)}{V_{0}}\right)^{2}, \quad \eta \simeq m_{\mathrm{P}}^{2} \frac{V^{\prime \prime}(\sigma)}{V_{0}},
$$

where the primes denote derivation with respect to the real normalized inflaton field $\sigma$ and $m_{\mathrm{P}} \simeq 2.44 \times 10^{18} \mathrm{GeV}$ is the reduced Planck scale. The conditions for inflation to take place are $\varepsilon \leq 1$ and $|\eta| \leq 1$.

Calculating the number of e-foldings $N_{Q}$ that our present horizon scale suffered during inflation, we obtain the following relation (see e.g. Ref. 43):

$$
N_{Q} \simeq \frac{1}{m_{\mathrm{P}}^{2}} \int_{\sigma_{f}}^{\sigma_{Q}} \frac{V_{0}}{V^{\prime}(\sigma)} d \sigma \simeq \ln \left(4.41 \times 10^{11} T_{r}^{\frac{1}{3}} V_{0}^{\frac{1}{6}}\right),
$$

where $\sigma_{f}\left[\sigma_{Q}\right]$ is the value of $\sigma$ at the end of inflation [when our present horizon scale crossed outside the inflationary horizon] and $T_{r} \simeq 10^{9} \mathrm{GeV}$ is the reheat temperature taken to saturate the gravitino constraint ${ }^{44}$.

The quadrupole anisotropy of the cosmic microwave background radiation can be calculated as follows (see e.g. Ref. 43):

$$
\left(\frac{\delta T}{T}\right)_{Q} \simeq \frac{1}{12 \sqrt{5}} \frac{V_{0}^{\frac{3}{2}}}{V^{\prime}\left(\sigma_{Q}\right) m_{\mathrm{P}}^{3}}
$$

Fixing $(\delta T / T)_{Q} \simeq 6.6 \times 10^{-6}$, which is its central value from the cosmic background explorer $(\mathrm{COBE})^{45}$ (assuming that the spectral index $n=1$ ), we can determine one of the free parameters (say $\beta$ ) in terms of the others $\left(m, \kappa\right.$ and $\lambda$ ). For instance, we find $\beta=0.1$, for $m=4.35 \times 10^{15} \mathrm{GeV}$ and $\kappa=\lambda=3 \times 10^{-2}$. In this case, the instability point of the shifted path lies at $\sigma_{c} \simeq 3.55 \times 10^{16} \mathrm{GeV}, \sigma_{f} \simeq 1.7 \times 10^{17} \mathrm{GeV}$ and $\sigma_{Q} \simeq 1.6 \times 10^{18} \mathrm{GeV}^{43}$. Also, $M \simeq 2.66 \times 10^{16} \mathrm{GeV}, N_{Q} \simeq 57.7$ and $n \simeq 0.98$. Note that the slow roll conditions are violated and, thus, inflation ends well before reaching the instability point at $\sigma_{c}$. We see that the COBE constraint can be easily satisfied with natural values of the parameters. Moreover, superheavy SM 
non-singlets with masses $\ll M_{\mathrm{GUT}}$, which could disturb the unification of the MSSM gauge coupling constants, are not encountered.

\subsection{Supergravity Corrections}

Here, inflation takes place at quite high $\sigma$ 's. So, supergravity (SUGRA) corrections are important and could easily invalidate inflation, in contrast to the standard hybrid inflation case, where they can be kept ${ }^{46}$ under control. This catastrophe can be avoided by invoking a specific Kähler potential (used in no-scale SUGRA models) and a gauge singlet $Z$ with a similar Kähler potential, as is suggested in Ref. 47. Assuming a superheavy VEV for $Z$ via D-terms, one can achieve an exact cancellation of the inflaton mass corrections on the shifted path. So, inflation remains intact, but gets considerably corrected via the kinetic terms of $\sigma$.

We find that, for the $\sigma$ 's under consideration, the SUGRA corrections have only a small influence on $\sigma_{Q}$ if we use the same input values for the free parameters as in the global SUSY case. On the contrary, $(\delta T / T)_{Q}$ increases considerably. However, we can easily readjust the parameters so that the COBE constraint is again met. For instance, $(\delta T / T)_{Q} \simeq 6.6 \times 10^{-6}$ is now obtained with $m=3.8 \times 10^{15} \mathrm{GeV}$ keeping $\kappa=\lambda=3 \times 10^{-2}, \beta=0.1$ as in global SUSY. In this case, $\sigma_{c} \simeq 2.7 \times 10^{16} \mathrm{GeV}, \sigma_{f} \simeq 1.8 \times 10^{17} \mathrm{GeV}$ and $\sigma_{Q} \simeq 1.6 \times 10^{18} \mathrm{GeV}$. Also, $M \simeq 2.6 \times 10^{16} \mathrm{GeV}, N_{Q} \simeq 57.5$ and $n \simeq 0.99$.

\section{Conclusions}

We have reviewed the construction of a SUSY GUT model based on the PS gauge group which naturally yields a YQUC, allowing an acceptable $b$-quark mass within the CMSSM with $\mu>0$. We found that there exists a wide and natural range of parameters consistent with the data on the CDM abundance in the universe, $b \rightarrow s \gamma$, the muon anomalous magnetic moment and the Higgs boson masses. Moreover, the model gives rise to a new version of the shifted hybrid inflationary scenario, which avoids overproduction of monopoles at the end of inflation by using only renormalizable interactions.

\section{Acknowledgments}

We would like to thank M.E. Gómez, R. Jeannerot and S. Khalil for fruitful and pleasant collaborations from which this work is culled. This work was supported by European Union under the RTN contracts HPRN-CT-200000148 and HPRN-CT-2000-00152. 


\section{References}

1. G.L. Kane, C. Kolda, L. Roszkowski and J.D. Wells, Phys. Rev. D 49, 6173 (1994) [hep-ph/9312272].

2. G. Lazarides and C. Panagiotakopoulos, Phys. Lett. B 337, 90 (1994) [hep-ph/9403316]; S. Khalil, G. Lazarides and C. Pallis, ibid. 508, 327 (2001) [hep-ph/0005021].

3. L. Hall, R. Rattazzi and U. Sarid, Phys. Rev. D 50, 7048 (1994) [hep-ph/ 9306309]; M. Carena, M. Olechowski, S. Pokorski and C.E.M. Wagner, Nucl. Phys. B426, 269 (1994) [hep-ph/9402253].

4. D. Pierce, J. Bagger, K. Matchev and R. Zhang, Nucl. Phys. B491, 3 (1997) [hep-ph/9606211].

5. M. Carena, D. Garcia, U. Nierste and C.E.M. Wagner, Nucl. Phys. B577, 88 (2000) [hep-ph/9912516].

6. S.F. King and M. Oliveira, Phys. Rev. D 63, 015010 (2001) [hep-ph/0008183].

7. S. Abel et al. (SUGRA Working Group Collaboration), hep-ph/0003154.

8. M.E. Gómez, G. Lazarides and C. Pallis, Nucl. Phys. B638, 165 (2002) [hep-ph/0203131].

9. H. Baer, J. Ferrandis, K. Melnikov and X. Tata, Phys. Rev. D 66, 074007 (2002) [hep-ph/0207126].

10. T. Blažek, R. Dermíšek and S. Raby, Phys. Rev. Lett. 88, 111804 (2002) [hep-ph/0107097]; Phys. Rev. D 65, 115004 (2002) [hep-ph/0201081].

11. D. Auto et. al., J. High Energy Phys. 06, 023 (2003) [hep-ph/0302155].

12. U. Chattopadhyay, A. Corsetti and P. Nath, Phys. Rev. D 66, 035003 (2002) [hep-ph/0201001]; C. Pallis, Nucl. Phys. B678, 398 (2004) [hep-ph/0304047].

13. G. Lazarides, Q. Shafi and C. Wetterich, Nucl. Phys. B181, 287 (1981); G. Lazarides and Q. Shafi, ibid. B350, 179 (1991).

14. M.E. Gómez, G. Lazarides and C. Pallis, Phys. Rev. D 67, 097701 (2003) [hep-ph/0301064]; C. Pallis and M.E. Gómez, hep-ph/0303098.

15. D. Spergel et al., Astrophys. J. Suppl. 148, 175 (2003) [astro-ph/0302209].

16. R. Barate et al. (ALEPH Collaboration), Phys. Lett. B 429, 169 (1998); K. Abe et al. (BELLE Collaboration), ibid. 511, 151 (2001) [hep-ex/0103042]; S. Chen et al. (CLEO Collaboration), Phys. Rev. Lett. 87, 251807 (2001) [hep-ex/0108032].

17. R. Jeannerot, S. Khalil and G. Lazarides, J. High Energy Phys. 07, 069 (2002) [hep-ph/0207244].

18. R. Jeannerot, S. Khalil, G. Lazarides and Q. Shafi, J. High Energy Phys. 10, 012 (2000) [hep-ph/0002151].

19. G. Lazarides, hep-ph/0011130; R. Jeannerot, S. Khalil and G. Lazarides, hep-ph/0106035.

20. I. Antoniadis and G.K. Leontaris, Phys. Lett. B 216, 333 (1989).

21. G. Lazarides and Q. Shafi, Phys. Rev. D 58, 071702 (1998) [hep-ph/9803397].

22. M.E. Gómez, G. Lazarides and C. Pallis, Phys. Rev. D 61, 123512 (2000) [hep-ph/9907261]; Phys. Lett. B 487, 313 (2000) [hep-ph/0004028].

23. M.E. Gómez and C. Pallis, hep-ph/0303094 (in the SUSY02 Proceedings).

24. J. Ellis, K.A. Olive, Y. Santoso and V.C. Spanos, Phys. Lett. B 565, 176 
(2003) [hep-ph/0303043]; A.B. Lahanas and D.V. Nanopoulos, ibid. 568, 55 (2003) [hep-ph/0303130]; H. Baer and C. Balázs, JCAP 05, 006 (2003) [hep-ph/0303114]; U. Chattopadhyay, A. Corsetti and P. Nath, Phys. Rev. D 68, 035005 (2003) [hep-ph/0303201].

25. H. Goldberg, Phys. Rev. Lett. 50, 1419 (1983); J.R. Ellis, J.S. Hagelin, D.V. Nanopoulos, K.A. Olive and M. Srednicki, Nucl. Phys. B238, 453 (1984).

26. G. Bélanger, F. Boudjema, A. Pukhov and A. Semenov, Comput. Phys. Commun. 149, 103 (2002) [hep-ph/0112278].

27. J. Ellis, T. Falk, K.A. Olive and M. Srednicki, Astropart. Phys. 13, 181 (2000), (E) ibid. 15, 413 (2001) [hep-ph/9905481].

28. A. Djouadi, J. Kalinowski and M. Spira, Comput. Phys. Commun. 108, 56 (1998) [hep-ph/9704448].

29. A.L. Kagan and M. Neubert, Eur. Phys. J. C 7, 5 (1999) [hep-ph/9805303];

P. Gambino and M. Misiak, Nucl. Phys. B611, 338 (2001) [hep-ph/0104034].

30. M. Ciuchini, G. Degrassi, P. Gambino and G. Giudice, Nucl. Phys. B527, 21 (1998) [hep-ph/9710335]; G. Degrassi, P. Gambino and G.F. Giudice, J. High Energy Phys. 12, 009 (2000) [hep-ph/0009337].

31. S. Martin and J. Wells, Phys. Rev. D 64, 035003 (2001) [hep-ph/0103067].

32. M. Davier, hep-ex/0312065 (to appear in the SIGHAD03 Proceedings).

33. G.W. Bennett et al. (Muon g-2 Collaboration), Phys. Rev. Lett. 89, 101804 (2002), (E) ibid. 89, 129903 (2002) [hep-ex/0208001].

34. ALEPH, DELPHI, L3 and OPAL Collaborations, The LEP Higgs working group for Higgs boson searches, hep-ex/0107029; LHWG-NOTE/2002-01, http://lephiggs . web. cern. ch/LEPHIGGS/papers/July2002_SM/index.html.

35. S. Heinemeyer, W. Hollik and G. Weiglein, hep-ph/0002213.

36. A.B. Lahanas, D.V. Nanopoulos and V.C. Spanos, Phys. Rev. D 62, 023515 (2000) [hep-ph/9909497].

37. A.D. Linde, Phys. Rev. D 49, 748 (1994) [astro-ph/9307002].

38. E.J. Copeland, A.R. Liddle, D.H. Lyth, E.D. Stewart and D. Wands, Phys. Rev. D 49, 6410 (1994) [astro-ph/9401011].

39. G. Lazarides and C. Panagiotakopoulos, Phys. Rev. D 52, 559 (1995) [hep-ph/9506325]; R. Jeannerot, S. Khalil and G. Lazarides, Phys. Lett. B 506, 344 (2001) [hep-ph/0103229].

40. T.W.B. Kibble, J. Phys. A 9, 1387 (1976).

41. G. Dvali, R. Schaefer and Q. Shafi, Phys. Rev. D 73, 1886 (1994) [hep-ph/ 9406319].

42. S. Coleman and E. Weinberg, Phys. Rev. D 7, 1888 (1973).

43. G. Lazarides, Lect. Notes Phys. 592, 351 (2002) [hep-ph/0111328]; hep-ph/ 0204294.

44. M.Yu. Khlopov and A.D. Linde, Phys. Lett. B 138, 265 (1984); J. Ellis, J.E. Kim and D.V. Nanopoulos, ibid. 145, 181 (1984).

45. C.L. Bennett et al., Astrophys. J. 464, L1 (1996) [astro-ph/9601067].

46. G. Lazarides, R.K. Schaefer and Q. Shafi, Phys. Rev. D 56, 1324 (1997) [hep$\mathrm{ph} / 9608256]$.

47. C. Panagiotakopoulos, Phys. Lett. B 459, 473 (1999) [hep-ph/9904284]. 